\title{
ESCOLHA ALIMENTAR DE UNIVERSITÁRIOS DE UMA UNIVERSIDADE DO PARANÁ
}

\section{CHOOSE FOOD OF UNIVERSITY STUDENTS FROM A UNIVERSITY OF PARANÁ}

\author{
Karine Aparecida de Lima', Kélin Schwarz ${ }^{2}$, Bruna Menegassi ${ }^{3}$, Tatiane Winkler \\ Marques Machado ${ }^{4}$, Paula Chuproski Saldan ${ }^{5}$
}

\begin{abstract}
1 - Graduada em Nutrição pela Universidade Estadual do Centro-Oeste
2 - Professora Doutora do Departamento de Nutrição da Universidade Federal do Triângulo Mineiro

3 - Professora Doutora da Universidade Federal da Grande Dourados

4 - Professora Doutora das Faculdades Integradas Espírita do Paraná

5 - Professora Doutora do Departamento de Nutrição da Universidade Estadual do Centro-Oeste Autor para correspondência: karyne_somer@yahoo.com.br
\end{abstract}

\section{RESUMO:}

O objetivo do estudo foi avaliar a escolha alimentar de usuários universitários beneficiados com o auxílio alimentação. Foi realizado um estudo transversal em uma Unidade Produtora de Refeição de um restaurante universitário da cidade de Guarapuava-PR, com 175 participantes. Foi avaliado o Índice de Massa Corporal (IMC) auto referido e a avaliação da escolha alimentar deu-se por meio de um cartão e do registro fotográfico dos pratos, para verificar a presença dos grupos de alimentos no almoço de cinco dias. De acordo com o IMC, a maior parte dos participantes encontrava-se em estado de eutrofia. Em relação a escolha alimentar, $96,5 \%(n=169)$ dos participantes escolheram alimentos do grupo dos cereais, raízes e tubérculos nos cinco dias avaliados, 60,5\% ( $n=106)$ escolheram alimentos do grupo das leguminosas (feijão) todos os dias da semana e $27,4 \% \quad(n=48)$ dos participantes escolheram esse alimento 3 a 4 vezes na semana, já a escolha de alimentos do grupo das hortaliças durante cinco dias da semana foi de $90,2 \%(n=158)$, do grupo das carnes $84,5 \%(n=148)$ dos participantes escolheram alimentos desse grupo todos os dias da semana e $11,4 \%(n=20)$ de 3 a 4 vezes. Em relação ao grupo de doces e açúcares aqui representado pelas sobremesas, $72,5 \%(n=127)$ dos participantes não escolheram alimentos desse grupo e $20,5 \%$ ( $n=36)$ escolheram 1 a 2 vezes na semana. A maioria dos participantes realizam escolhas saudáveis, o que mostra que o auxílio alimentação tem sido um ponto positivo para a alimentação dos comensais beneficiados.

Palavras-Chave: Alimentos. Índice de Massa Corporal. Restaurantes. Universidades.

\section{ABSTRACT:}

The objective of the study was to evaluate the food choice of university users benefit from the food assistance. This cross-sectional study was conducted in a Unit Meal Producer of a university restaurant in the city of Guarapuava-PR, Brazil, with 175 participants. The Body Mass Index (BMI) self-reported was evaluated, and the assessment of food choice was through a card and photographic record of the dishes, to analyze the presence of the food groups at lunch for five days. According to the BMI, the greater part of the participants were in eutrophic state. Regarding to food choice, $96.5 \%(n=169)$ of participants food choice of grains, roots and tubers on five days evaluated, 60.5\% ( $n=106)$ consumed the food of legumes group foods (beans) every day of the week and $27.4 \%(n=48)$ of the participants food choice this group 3-4 times a week. The food choice group of vegetables for 5 days of the week was $90.2 \%(n=158)$, the group of meat $84.5 \%(n=148)$ of participants food choice every day of the week and $11.4 \%(n=20)$ of 3-4 times a week. Regarding candies and sugar 
group represented by desserts, $72.5 \%(n=127)$ of participants did not choose food and $20.5 \%(n=36)$ food choice 1-2 times a week. The majority of participants perform healthy choices, which shows that food aid has been a plus point for the feeding of benefit dinners. Key words: Food. Body Mass Index. Restaurants. Universities.

\section{INTRODUÇÃO}

A escolha alimentar da população brasileira vem sofrendo modificações nos últimos anos devido mudanças estruturais, econômicas, demográficas, entre outras (PINHO et al., 2012). As escolhas alimentares cotidianas das pessoas ocorrem pela influência de fatores que vão além das condições de vida, como renda, urbanização e acesso à variedade de alimentos, e incluem também características individuais como educação, faixa etária, cultura alimentar e familiar (MACIEL et al., 2012).

Ao ingressar na universidade, o estudante passa por mudanças e adquire responsabilidades novas e isso inclui a alimentação. Portanto, o comportamento alimentar desses jovens vai depender de fatores como tempo, localização disponível para alimentação, condição financeira, psicológica e disponibilidade de alimentos locais; fatores que podem gerar riscos à saúde (SANTOS et al., 2014).

Nesse contexto, os restaurantes universitários devem proporcionar uma alimentação adequada aos comensais, e isso é possível quando a Unidade Produtora de Refeições (UPR) universitária conhece o perfil da clientela atendida, o seu estado nutricional e o número de refeições diárias necessárias para atender esse público. Se essas informações não são conhecidas estima-se o perfil e as necessidades dos comensais, mas isso impossibilita verificar o impacto da alimentação e segurança alimentar oferecidas (FAUSTO et al., 2001).

O auxílio alimentação oferecido pela universidade tem a função de proporcionar uma refeição com um custo mais acessível aos alunos, e sem a necessidade de deslocamento da universidade, pois o mesmo é válido no próprio restaurante universitário, o que reduz parte dos custos com alimentação e locomoção até outros locais. O mesmo também contribui no quesito tempo, pois os beneficiados não precisam sair da universidade para encontrar outros locais que proporcionem uma alimentação a um custo acessível.

A aplicação de uma proposta rápida e prática para a avaliação da escolha alimentar baseada em observação e registro dos grupos de alimentos presentes nos pratos dos usuários pode ser útil nesse sentido e, portanto, o objetivo desse estudo foi avaliar a escolha alimentar de usuários de uma UPR, beneficiados por auxílio alimentação. 


\section{MATERIAL E MÉTODOS}

Trata-se de um estudo transversal que foi realizado em uma UPR de um restaurante universitário da cidade de Guarapuava-PR, a qual possui um profissional nutricionista responsável técnico e usuários assíduos e que são beneficiados por auxílio alimentação oferecido por uma universidade. O estudo ocorreu nos meses de novembro e dezembro de 2015, considerando as fases de planejamento, execução e avaliação, contudo a coleta dos dados propriamente dita restringiu-se a 5 dias da semana.

O número de usuários inscritos que recebiam o benefício do auxílio alimentação para a referida UPR era de 692 no período do estudo. O cálculo amostral foi realizado no programa estatístico OpenEpi (DEAN; SULLIVAN; SOE, 2015). O número de usuários para compor a amostra do presente estudo foi de 248, com base na frequência antecipada de $50 \%$, por se tratar de um estudo exploratório, e precisão de 5\%. Os critérios de inclusão do presente estudo foram: usuários maiores de 18 anos que realizavam o almoço no restaurante universitário e que eram beneficiados pelo auxílio alimentação. Os critérios de exclusão foram usuários não beneficiados pelo auxílio alimentação e menores de 18 anos.

A avaliação da escolha alimentar dos usuários da UPR se deu com base nos grupos alimentares presentes no prato de cada usuário. Durante cinco dias consecutivos de uma mesma semana (segunda à sexta-feira), registraram-se os grupos alimentares presentes no prato de cada usuário em um cartão individual (Figura 1) identificando o grupo dos Cereais, raízes e tubérculos com a letra $A$, Leguminosas com a letra $B$, Frutas com a letra C, Hortaliças com a letra D, Carnes com a letra E, neste grupo foram inclusos os ovos, pois foram considerados alimentos do grupo das carnes e o grupo dos Doces e Açúcares foi identificado com a letra F.

\begin{tabular}{|c|c|c|c|c|c|c|c|c|c|c|c|c|c|c|c|c|c|c|c|c|c|c|c|c|c|c|c|c|c|c|c|}
\hline \multicolumn{32}{|c|}{$\begin{array}{l}\text { Cartāo de avaliação de escolha alimentar } \\
\text { (a ser preenchido pelo pesquisador) }\end{array}$} \\
\hline \multicolumn{32}{|c|}{ Nome: } \\
\hline \multicolumn{5}{|c|}{ Segunda } & \multicolumn{5}{|c|}{ Terça } & \multicolumn{6}{|c|}{ Quarta } & \multicolumn{5}{|c|}{ Quinta } & \multicolumn{5}{|c|}{ Sexta } & & \multicolumn{5}{|c|}{ Total } \\
\hline $\bar{A} \mid \mathrm{E}$ & C & D & E & $F$ & A & B & $\mathrm{C} \mid \mathrm{D}$ & $\mathrm{E}$ & $\mathrm{F}$ & A & $B$ & C & D) & $E$ & $\mathrm{~F}$ & A & \begin{tabular}{|l|l|}
$B$ & $C$ \\
\end{tabular} & 0 & $E$ & $F$ & A & $B$ & C.1 & $\mathrm{D}$ & $\begin{array}{ll}E & \end{array}$ & $F$ & & $3 \mathrm{C}$ & D & E & $\mathrm{F}$ \\
\hline & & & & & & & & & & & & & & & & & & & & & & & & & & & & & & 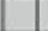 & \\
\hline
\end{tabular}

FIGURA 1 - Cartão de avaliação de escolha alimentar dos participantes do estudo, Guarapuava-PR, 2015

A escolha desses grupos de alimentos deve-se ao fato de a maioria deles representarem 
alimentos de um prato saudável e fazerem alusão ao Programa "Meu Prato Saudável" proposto por pesquisadores do Hospital das Clínicas e do Instituto do Coração da Faculdade de Medicina da Universidade de São Paulo (SERVIÇO DE NUTRIÇÃO DO INSTITUTO DO CORAÇÃO DO HC-FMUSP; LATINMED, 2014). A fim de assegurar a correta avaliação dos grupos de alimentos, o prato, juntamente com o cartão que continha informações sobre o prato do participante, foram fotografados ao final do balcão das preparações e o pesquisador pôde conferir detalhadamente os grupos presentes nos pratos dos comensais participantes do estudo (Figura 2).

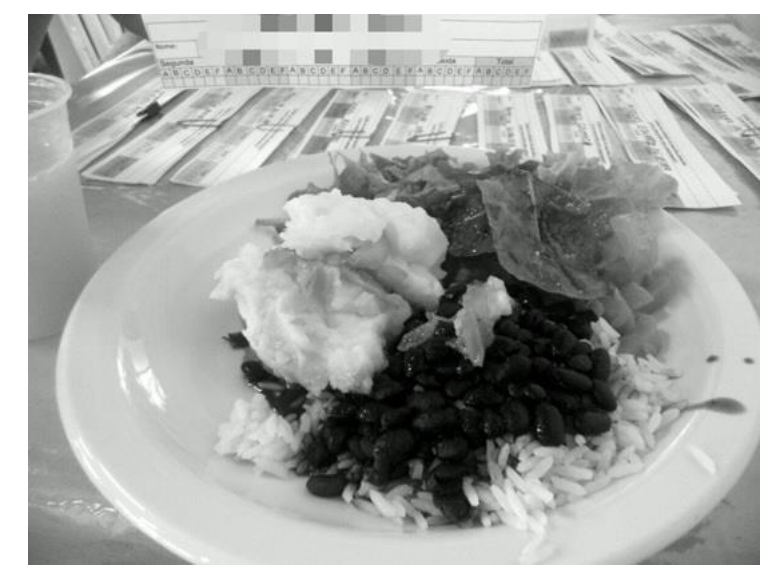

FIGURA 2 - Foto do prato de um participante do estudo, Guarapuava-PR, 2015

Os dados coletados foram tabulados no Programa Microsoft Excel® e foram analisados por meio de estatística descritiva no programa Stata 12.0 (StataCorp, College Station, Texas, EUA). Foi calculado o Índice de Massa Corporal (IMC) dos usuários com base no peso e estatura referidos, solicitados no momento da inscrição, e classificados de acordo com os seguintes pontos de corte: Magreza Grau III <16 kg/m2, Magreza grau II 16 a 16,9 kg/m2, Magreza grau I 17 a 18 kg/m2, Eutrofia 18,5 a 24,9 kg/m2, Sobrepeso 25 a $24,9 \mathrm{~kg} / \mathrm{m} 2$, Obesidade grau I 30 a $34,9 \mathrm{~kg} / \mathrm{m} 2$, Obesidade grau II 35 a $40 \mathrm{~kg} / \mathrm{m} 2$, Obesidade grau III > 40 kg/m2 (WORLD HEALTH ORGANIZATION, 1995; 2000).

Os resultados da escolha alimentar dos usuários da UPR foram apresentados de forma descritiva e analisados para o conjunto dos cinco dias da semana, período no qual foi realizado o estudo. Para avaliar a frequência (cinco dias) da escolha alimentar estabeleceu-se a seguinte padronização: de 1 a 2 vezes na semana: escolha esporádica; de 3 a 4 vezes: escolha regular; 5 vezes: escolha diária e, quando não houve escolha de determinado alimento no dia, considerou-se que não houve escolha.

Os participantes foram esclarecidos sobre o estudo e aqueles que manifestaram 
interesse em participar assinaram o Termo de Consentimento Livre e Esclarecido e preencheram uma ficha com informações pessoais sobre idade, peso e estatura referidos. O estudo foi aprovado pelo Comitê de Ética em Pesquisa de uma Instituição de Ensino Superior do Estado do Paraná (Parecer № 1.202.329 de 09/10/2015) e seguiu a Resolução do Conselho Nacional de Saúde № 466/2012.

\section{RESULTADOS E DISCUSSÃO}

Da amostra calculada, 175 (68\%) usuários da UPR aceitaram participar do estudo e houve a recusa de 82 (32\%). A recusa pode ser devido informações obtidas junto a estes que alegavam não almoçar com frequência no restaurante universitário ou utilizavam o auxílio alimentação para comprar lanches. Outro motivo que pode explicar essa recusa refere-se ao desconforto por parte do usuário de ter seu prato fotografado por cinco dias.

Dos participantes do estudo, 126 (72\%) eram do sexo feminino e 49 (28\%) do sexo masculino, com idade média de 21 anos (desvio padrão=2,6), sendo a idade mínima 18 e a máxima de 34 anos. O IMC médio foi de $22,9 \mathrm{~kg} / \mathrm{m}^{2}$ (desvio padrão=3,4), sendo o IMC mínimo de $16,2 \mathrm{~kg} / \mathrm{m}^{2}$ e o máximo de $34,1 \mathrm{~kg} / \mathrm{m}^{2}$. Na Tabela 1 está apresentada a classificação do IMC dos participantes avaliados.

TABELA 1. AVALIAÇÃO DO IMC DE USUÁRIOS UNIVERSITÁRIOS BENEFICIADOS COM O AUXÍLIO ALIMENTAÇÃO DE UMA UPR DE GUARAPUAVA-PR, 2015

\begin{tabular}{lcc}
\hline Classificação do IMC & $\mathbf{n}$ & Percentual (\%) \\
\hline Magreza grau II & 3 & 1,7 \\
Magreza grau I & 2 & 1,1 \\
Eutrofia & 128 & 73,1 \\
Sobrepeso & 34 & 19,4 \\
Obesidade grau I & 8 & 4,6 \\
\hline
\end{tabular}

$\mathrm{IMC}=$ Índice de Massa Corporal, UPR= Unidade Produtora de Refeições.

Pode-se observar que a maioria dos estudantes se encontrava eutrófica no período do estudo. Resultado similar foi reportado por Kretschmer et al. (2015) que avaliaram o estado nutricional e hábitos alimentares de acadêmicos de uma universidade e encontraram $71,2 \%$ dos participantes em estado de eutrofia. Oliveira, Guaglianoni e Demonte (2005) ao avaliarem indivíduos adultos 
usuários de restaurante universitário encontraram que 74,7\% dos indivíduos estavam eutróficos.

$\mathrm{Na}$ tabela 2 estão apresentados os resultados referentes a escolha alimentar, 96,5\% ( $n=169)$ dos participantes escolheram alimentos do grupo dos Cereais, raízes e tubérculos nos 5 dias avaliados, 60,5\% ( $n=106)$ escolheram alimentos do grupo das Leguminosas (feijão) todos os dias da semana e $27,4 \% \quad(n=48)$ dos participantes consumiram esse alimento 3 a 4 vezes na semana.

TABELA 2. ESCOLHA E FREQUÊNCIA ALIMENTAR DE USUÁRIOS UNIVERSITÁRIOS BENEFICIADOS COM O AUXÍLIO ALIMENTAÇÃO DE UMA UPR DE GUARAPUAVA-PR, 2015

\begin{tabular}{|c|c|c|}
\hline Grupos/Frequência & $\mathbf{n}$ & Percentual (\%) \\
\hline \multicolumn{3}{|l|}{ Grupo A } \\
\hline Escolha Diária & 169 & 96,5 \\
\hline Escolha Regular & 3 & 1,7 \\
\hline Escolha Esporádica & 0 & 0 \\
\hline Não houve escolha & 3 & 1,7 \\
\hline \multicolumn{3}{|l|}{ Grupo B } \\
\hline Escolha Diária & 106 & 60,5 \\
\hline Escolha Regular & 48 & 27,4 \\
\hline Escolha Esporádica & 14 & 8,0 \\
\hline Não houve escolha & 7 & 4,0 \\
\hline \multicolumn{3}{|l|}{ Grupo D } \\
\hline Escolha Diária & 158 & 90,2 \\
\hline Escolha Regular & 16 & 9,1 \\
\hline Escolha Esporádica & 0 & 0 \\
\hline Não houve escolha & 1 & 0,5 \\
\hline \multicolumn{3}{|l|}{ Grupo E } \\
\hline Escolha Diária & 148 & 84,5 \\
\hline Escolha Regular & 20 & 11,4 \\
\hline Escolha Esporádica & 5 & 2,8 \\
\hline Não houve escolha & 2 & 1,4 \\
\hline \multicolumn{3}{|l|}{ Grupo F } \\
\hline Escolha Diária & 5 & 2,8 \\
\hline Escolha Regular & 7 & 4,0 \\
\hline Escolha Esporádica & 36 & 20,5 \\
\hline Não houve escolha & 127 & 72,5 \\
\hline
\end{tabular}


A escolha de alimentos do grupo das Hortaliças durante cinco dias da semana foi de $90,2 \%(n=158)$. Em relação ao grupo das Carnes, $84,5 \%(n=148)$ dos participantes escolheram todos os dias da semana e 11,4\% $(n=20)$ de 3 a 4 vezes por semana. Quanto à escolha do grupo de Doces e açúcares aqui representado pelas sobremesas, 72,5\% $(n=127)$ dos participantes não escolheram e 20,5\% ( $n=36)$ escolheram 1 a 2 vezes na semana. A escolha de alimentos do grupo das Frutas não esteve presente em nenhum dia avaliado devido ao restaurante não ter a opção de frutas no cardápio.

Destacam-se como dados positivos desse estudo a escolha alimentar de alimentos básicos e in natura como arroz, feijão, carnes e hortaliças, o que contradiz Ramalho, Dalamaria e Souza (2012) que por meio de um estudo transversal avaliaram 863 universitários os quais apresentaram baixo percentual de escolha de hortaliças $(14,8 \%)$. Vieira, Sabadin e Oliveira (2008) também avaliaram o estado nutricional e as práticas alimentares de universitários e observaram percentual de $30 \%$ de usuários que não consumiam hortaliças. Visto a comparação com esses dados da literatura, os resultados do presente estudo precisam ser analisados com cautela, uma vez que os participantes que aceitaram participar do estudo poderiam apresentar melhor hábito alimentar comparados com aqueles que não aceitaram participar.

Ressalta-se como dado negativo desse estudo a ausência de frutas no cardápio da UPR como opção de sobremesa ou como componente das saladas e, a oferta de sobremesas, geralmente ricas em açúcar, como gelatina e sagu. Assim a baixa escolha de sobremesa doce observada no estudo, pode não representar o hábito dos comensais, já que existiu baixa oferta, sendo que somente os primeiros comensais a se servir tinham acesso a opção de sobremesa. Então a baixa escolha de alimentos do grupo dos Doces e açúcares (escolha diária e regular=6,8\%), pode ter ocorrido devido à baixa disponibilidade de sobremesas no restaurante, e também pelo fato da sobremesa quase sempre se repetir, não havendo variações.

Dados divergentes foram encontrados pelo VIGITEL (Vigilância de fatores de risco e proteção para doenças crônicas por inquérito telefônico), que ao avaliar a escolha de doces durante cinco ou mais dias da semana em adultos encontrou maior escolha de doces e açúcares na população avaliada, cerca de 18,1\% (BRASIL, 2015).

De acordo com Santos et al. (2011), os restaurantes por peso oferecem a facilidade de uma refeição completa com um custo acessível ao comensal, quando comparados a outros serviços de alimentação fora de casa. Isso possibilita o comensal fazer as escolhas alimentares que vão compor seu prato de acordo com suas preferências e hábitos 
alimentares. Os comensais que não participaram do estudo ou que não utilizavam o auxílio alimentação para se alimentar no balcão de preparações por quilo da UPR e compravam lanches poderiam ter uma escolha alimentar menos saudável com a escolha de alimentos fritos, ricos em gorduras, sódio e açúcares, porém isso não pode ser afirmado já que não foi objeto do presente estudo.

A alimentação de $90 \%$ dos brasileiros é rica em produtos calóricos e de baixo teor nutritivo. Essa foi a conclusão da Análise de Consumo Alimentar Pessoal no Brasil, da Pesquisa de Orçamentos Familiares (POF), realizada pelo Instituto Brasileiro de Geografia e Estatística (IBGE) em 2008/2009 (INSTITUTO BRASILEIRO DE GEOGRAFIA E ESTATÍSTICA, 2011). Porém dados encontrados no presente estudo revelaram situação diferente, pois entre os participantes observou-se escolha diária de Cereais, raízes e tubérculos por 169 participantes (96,5\%), Leguminosas, 106 (60,5\%), Hortaliças, 158 $(90,2 \%)$ e Carnes, $148(84,5 \%)$, resultado similar ao de Carvalho e Rocha (2011), que avaliaram a alimentação a partir de um Questionário de Frequência Alimentar (QFA), em um estudo transversal, com um público composto por 150 indivíduos adultos e encontraram alto percentual de escolha do grupo dos cereais $(n=150,100 \%)$, leguminosas $(n=141,94 \%)$, carnes $(n=111,74 \%)$ e hortaliças ( $n=129,86 \%)$.

Os dados encontrados em relação ao IMC e as escolhas alimentares foram positivos, pois a maior parte dos participantes além de eutróficos, realizam escolhas alimentares saudáveis, mostrando que o auxílio alimentação tem contribuído beneficamente para a manutenção do estado nutricional dos participantes. No entanto, sugere-se que haja oferta de frutas da época como opção de sobremesa, pois além de terem um custo acessível, a unidade estaria proporcionando uma refeição mais equilibrada e saudável aos comensais.

O presente estudo apresenta algumas limitações, entre elas podemos destacar que a avaliação da escolha alimentar foi apenas qualitativa e não quantitativa e o fato de fotografar o prato pode ter alterado os alimentos normalmente consumidos pelos comensais. Além disso, não foi avaliado a escolha alimentar em outros momentos do dia, como no jantar e em casa, que pode ser distinto do momento do almoço no restaurante universitário. Também merece destaque o fato de o estudo ter se restringido a cinco dias consecutivos da semana que pode ter modificado o hábito dos comensais já que sabiam que estavam sendo avaliados 


\section{REFERÊNCIAS}

BRASIL. Ministério da Saúde. VIGITEL Brasil 2014: vigilância de fatores de risco e proteção para doenças crônicas por inquérito telefônico. Brasília: Ministério da Saúde, 2015.

CARVALHO, E. O.; ROCHA, E. F. Consumo alimentar de população adulta residente em área rural da cidade de lbatiba (ES, Brasil). Ciência \& Saúde Coletiva, Rio de Janeiro, v. 16, n. 1, p. 179-185, jan. 2011.

DEAN, A. G.; SULLIVAN, K. M.; SOE, M. M. OpenEpi: Open Source Epidemiologic Statistics for Public Health. Versão 3.01 Disponível em: www.OpenEpi.com. Acesso em: 10 jun. 2015.

FAUSTO, M. A. et al. Determinação do perfil dos usuários e da composição química e nutricional da alimentação oferecida no restaurante universitário da universidade estadual paulista, Araraquara, Brasil. Revista de Nutrição, Campinas, v. 14, n. 3, p. 171-176, 2001.

INSTITUTO BRASILEIRO DE GEOGRAFIA E ESTATístICA. Pesquisa de Orçamentos Familiares (POF 2008-2009): análise do consumo alimentar pessoal no Brasil. IBGE: Rio de Janeiro, 2011.

KRETSCHMER, A. C. et al. Estado nutricional e hábitos alimentares de acadêmicos de uma universidade do norte do Rio Grande do Sul. Revista Saúde, Santa Maria, v. 41, n. 2, p. 121-128, 2015.

MACIEL, E. S. et al. Consumo alimentar, estado nutricional e nível de atividade física em comunidade universitária brasileira. Revista de Nutrição, Campinas, v. 6, n. 25, p. 707-718, 2012.

OLIVEIRA, R. B.; GUAGLIANONI, D. G.; DEMONTE, A. Perfil do usuário, composição e adequação nutricional do cardápio oferecido em um restaurante universitário. Alimentos e Nutrição, Araraquara, v. 16, n. 4, p. 397-401, 2005.

PINHO, C. P. S. et al. Consumo de alimentos protetores e preditores do risco cardiovascular em adultos do estado de Pernambuco. Revista de Nutrição, Campinas, v. 25, n. 3, p. 341351, 2012. 
RAMALHO, A. A.; DALAMARIA, T.; SOUZA, O. F. Consumo regular de frutas e hortaliças por estudantes universitários em Rio Branco, Acre, Brasil: prevalência e fatores associados. Cadernos de Saúde Pública, Rio de Janeiro, v. 28, n. 7, p. 1405-13, 2012.

SANTOS, A. K. G. V. et al. Qualidade de vida e alimentação de estudantes universitários que moram na região central de São Paulo sem a presença dos pais ou responsáveis. Revista Simbio-Logias, Botucatu, v. 7, n. 10, p. 76-99, 2014.

SANTOS, M. V. et al. Os restaurantes por peso no contexto de alimentação saudável fora de casa. Revista de Nutrição, Campinas, v. 24, n. 4, p. 641-649, 2011.

SERVIÇO DE NUTRIÇÃO DO INSTITUTO DO CORAÇÃO DO HC-FMUSP; LATINMED. Meu Prato Saudável. São Paulo: ELM Marketing e editora médica, 2014.

VIEIRA, C. M.; SABADIN, E.; OLIVEIRA, M. R. M. Avaliação das práticas alimentares e do estado nutricional de universitárias do primeiro ano de nutrição. Revista Simbio-Logias, Botucatu, v. 1, n. 1, p. 87-98, 2008.

WORLD HEALTH ORGANIZATION. Physical status: the use and interpretation of anthropometry. Geneva: World Health Organization, 1995.

WORLD HEALTH ORGANIZATION. Obesity: preventing and managing the global epidemic of obesity. Geneva: World Health Organization, 2000. 